\title{
Trans-Spinal Direct Current Stimulation Alters Muscle Tone in Mice with and without Spinal Cord Injury with Spasticity
}

\author{
Zaghloul Ahmed \\ Department of Physical Therapy, College of Staten Island for Developmental Neuroscience, the College of Staten Island, Staten Island, New York 10314, and \\ Graduate Center/The City University of New York, New York, New York 10016
}

\begin{abstract}
Muscle tone abnormalities are associated with many CNS pathologies and severely limit recovery of motor control. Muscle tone depends on the level of excitability of spinal motoneurons and interneurons. The present study investigated the following hypotheses: (1) direct current flowing from spinal cord to sciatic nerve [spinal-to-sciatic direct current stimulation (DCS)] would inhibit spinal motor neurons and interneurons, hence reducing muscle tone; and (2) direct current flowing in the opposite direction (sciatic-to-spinal DCS) would excite spinal motor neurons and interneurons, hence increasing muscle tone. Current intensity was biased to be $\sim 170$ times greater at the spinal column than at the sciatic nerve. The results showed marked effects of DCS on muscle tone. In controls and mice with spinal cord injuries with spasticity, spinal-to-sciatic DCS reduced transit and steady stretch-induced nerve and muscle responses. Sciatic-to-spinal DCS caused opposite effects. These findings provide the first direct evidence that trans-spinal DCS can alter muscle tone and suggest that this approach could be used to reduce both hypotonia and hypertonia.
\end{abstract}

Key words: direct current; sciatic nerve; spasticity; spinal cord

\section{Introduction}

Management of muscle tone abnormalities is a serious and sometimes insurmountable challenge. Many CNS disorders involve an increase (hypertonus) or decrease (hypotonus) in muscle tone. For example, hypotonia is commonly observed in cerebellar deficits (Gilman, 1969), spinocerebellar lesions (Subramony and Ashizawa, 1993), and developmentally delayed children (Shumway-Cook and Woollacott, 1985). Hypertonia is associated with many CNS disorders, including stroke (Burke et al., 2013) and spinal cord injury (SCI; Adams and Hicks, 2005). Hypertonia includes spasticity and rigidity, and is characterized by a velocity-dependent increase in tonic stretch reflexes (Lance, 1980) and increased muscle activity during passive stretch (Katz and Rymer, 1989). Spasticity can range from mild to severe and can cause striking impairments in functional movement (Adams and Hicks, 2005). Pharmacological, surgical, and physical treatments to manage spasticity have at best short-term efficacy and are confounded by side effects (Gracies et al., 1997).

Increases in reflex excitability following spinal cord injury may be caused by a number of factors, including increased excitability of

Received 0ct. 17, 2013; revised Dec. 15, 2013; accepted Dec. 18, 2013.

Author contributions: Z.A. designed research; Z.A. performed research; Z.A. contributed unpublished reagents/ analytic tools; Z.A. analyzed data; Z.A. wrote the paper.

This work was supported by the New York State Department of Health/Wadsworth Center/Spinal Cord Injury Research Board. I thank Professor Andrzej Wieraszko for helpful comments on early version of this manuscript. I also thank Alzahraa Amer for technical assistance.

CUNY has filed a patent application based on the content of this study. The author declares no other competing financial interests.

Correspondence should be addressed to Zaghloul Ahmed, Department of Physical Therapy, College of Staten Island for Developmental Neuroscience, the College of Staten Island, Staten Island, NY 10314. E-mail: zaghloul.ahmed@csi.cuny.edu.

DOI:10.1523/JNEUROSCI.4445-13.2014

Copyright $\odot 2014$ the authors $\quad 0270-6474 / 14 / 341701-09 \$ 15.00 / 0$ spinal motoneurons (Cope et al., 1986; Bennett et al., 2001; Heckmann et al., 2005), and changes in interneuronal physiology (Hornby et al., 2003; Onushko et al., 2011) and connectivity (Yates et al., 2009; Kapitza et al., 2012). In general, following spinal cord injury, increased excitation and reduced inhibition of the mechanisms controlling motoneurons causes abnormal generation of force (Roy and Edgerton, 2012). We previously showed that dorsal surface anodal stimulation of the spinal cord decreases spinal excitability, while cathodal stimulation increases excitability (Ahmed, 2011). This agrees with previous studies, despite differences in location of the return electrode (Fuortes, 1954; Eccles et al., 1962). Recently, we showed that trans-spinal direct current stimulation (DCS) modulates spinal interneuron excitability (Ahmed, 2013b). In addition, trans-spinal DCS had previously been shown to modulate the excitability of primary afferent fibers via their presynaptic terminals (Eccles et al., 1962). These findings provide the basis for clinical trans-spinal DCS applications in disorders with maladaptive excitation-inhibition balance (e.g., spasticity). Thus, spinal DCS has been proposed to treat spasticity (Elbasiouny et al., 2010), but it remains unknown what underlying mechanisms or direction of current could modulate the neural mechanisms controlling muscle tone.

In the present study, it was hypothesized that (1) direct current flowing from the dorsum of the spinal cord to abdominal skin and sciatic nerve would reduce spinal cord excitability, thereby reducing muscle tone; and (2) direct current flowing in the opposite direction would increase spinal cord excitability, thereby increasing muscle tone. In principle, the stimulation technique presented here was designed to modulate the background activity level of the motoneuron pool of the muscle and to change the firing threshold of motoneurons.

In the present study, nerve discharges and muscle resistance were measured during passive muscle stretches. Supporting the 
hypotheses, in healthy mice and in mice with clinical signs of spasticity following spinal cord injury, nerve discharges and muscle resistance during transit and steady-state muscle stretches were reduced by current flowing from the dorsal surface of the spinal cord to the periphery and were increased by current flowing in the opposite direction.

\section{Materials and Methods}

Animals

Adult male CD-1 mice ( $n=42$; weight, $35-40$ g) were used for this study. Protocols were approved by the Institutional Animal Care and Use Committee of the College of Staten Island.

\section{Spinal cord contusion injury}

Mice were deeply anesthetized with ketamine/ xylazine (90/10 mg/kg, i.p.), and a spinal contusion was produced using a Multicenter Animal Spinal Cord Injury Study/New York University impactor via an impact rod (1-mmdiameter head, $5.6 \mathrm{~g}$ ) released from a distance of $6.25 \mathrm{~mm}$ onto the T13 spinal cord level, exposed by a T10 laminectomy. Animals were tested 4 weeks after spinal cord injury.

\section{Experimental procedures}

The general procedure has been used previously by our laboratory (Ahmed, 2011, 2013a; Ahmed and Wieraszko, 2012), and the experimental setup is shown in Figure 1A. Animals were anesthetized using ketamine/xylazine (90/10 mg/kg, i.p.). Throughout experiments, anesthesia was kept at a moderate level, which was monitored on-line by observing muscle and nerve activity (Ahmed, 2013a). Animals were placed in a mouse stereotaxic apparatus, which was placed in a custom-made clamping spinal column system. The bone at the base of the tail was fixed to the base of the system with surgical pins. Holes were made at the distal parts of the femur and tibia bones, and nails were inserted to fix these bones to the base. Incisions were made in the skin covering the vertebral column (from midthoracic to sacral region) and hindlimb, and the skin was moved to the side and held with clips. The triceps surae (TS) muscle was carefully separated from the surrounding tissue. The tendon of the TS was connected to a force transducer that was connected to a bridge amplifier (ADInstruments). Tissue surrounding the distal part of the sciatic nerve was removed. Both the sciatic nerve and TS muscle were covered with a mixture of silicone oil and petroleum jelly (Vaseline).

DCS setup. A breadboard was used to design a current divider. A Grass stimulator with current isolation unit (DC mode) was used as the current source. As shown in Figure 1A, the anode was split into two branches. One branch (a1) was directly connected to a stainless steel plate electrode (thickness, $50 \mu \mathrm{m}$; width, $5 \mathrm{~mm}$; length, $7 \mathrm{~mm}$ ). The electrode was covered with 1 -mm-thick wick fabric (soaked with $0.9 \%$ saline) and placed over the spinal column. A second branch (a2) was passed through a 10 $\mathrm{M} \Omega$ resistor, then connected to a stainless steel plate electrode (width, 5 $\mathrm{mm}$ ), which was placed under the sciatic nerve. The cathode was split into two branches: one branch (c1) was directly connected to an alligator clip electrode attached to abdominal skin, and the second branch (c2)
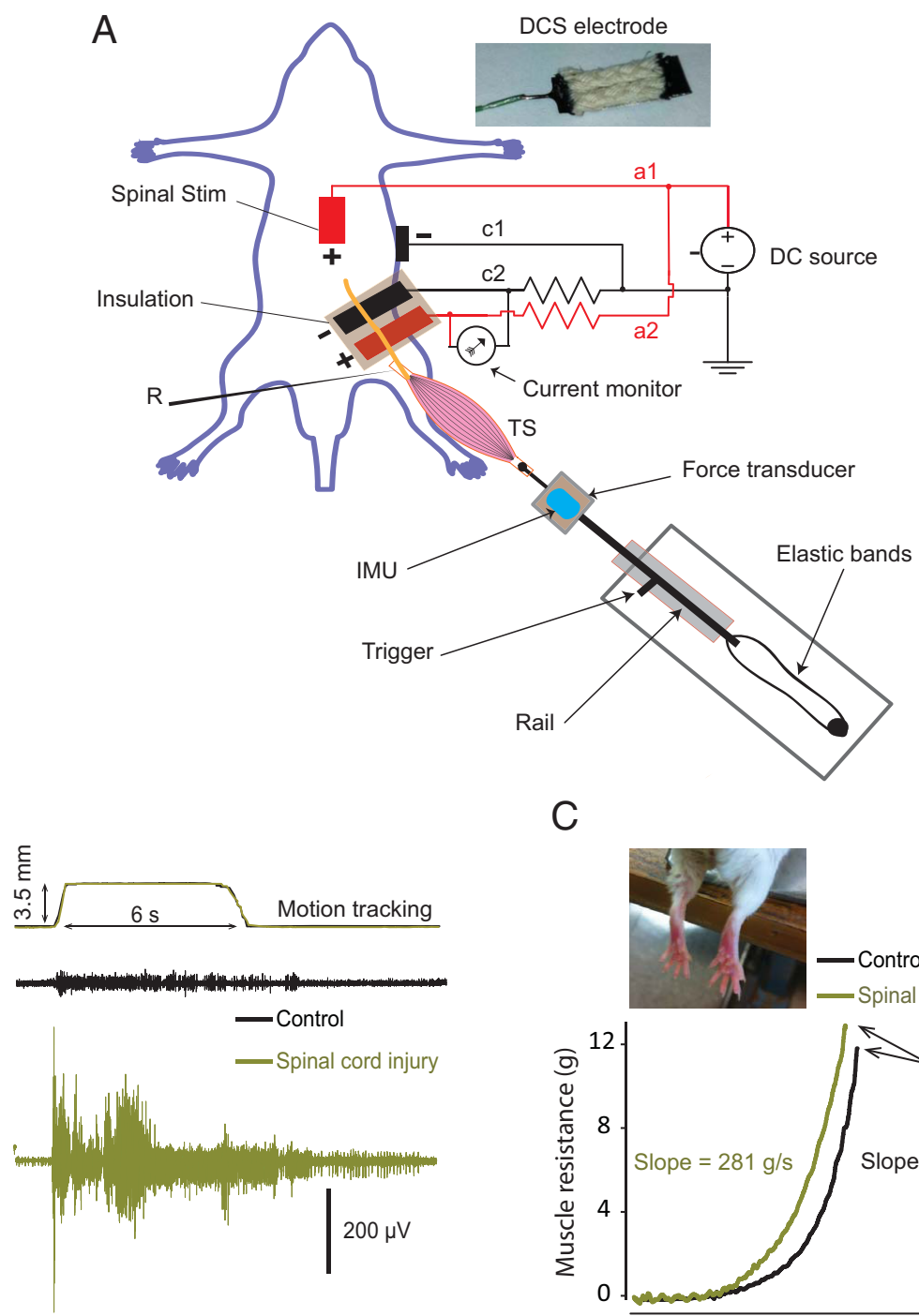

C
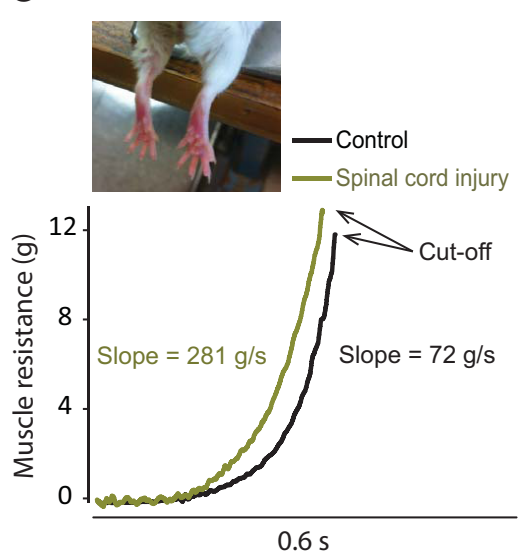

Figure 1. A, Experimental setup to stretch the TS muscle, record tibial nerve compound action potential (R), and deliver spinal-to-sciatic DCS. The anode $(+)$ is split into a1, which carries unattenuated current to the thoracolumbar spinal column, and a2, which carries attenuated current through a $10 \mathrm{M} \Omega$ resistor to a stainless steel electrode plate. The cathode was divided into $\mathrm{c} 1$, which carries unattenuated current to the abdominal skin, and c2, which carries attenuated current through a $10 \mathrm{M} \Omega$ resistor to 作 the base. A force transducer and IMU are fixed to the carriage. The carriage can be stopped at any position by a handle (trigger). Inset (top) shows tsDC electrode, covered with wick fabric. $\boldsymbol{B}$, Top, A recording (two traces) from the IMU tracking the movement stretch apparatus, showing that the stretch apparatus movements are identical. The bottom two panels show samples of stretch responses recorded from the tibial nerve in control (black) and SCl animals (green). Note that no DCS was applied during these recordings. C, Two examples of reflex force traces were recorded by the force transducer during $209 \mathrm{~mm} / \mathrm{s}$ lengthening (b) initiation) is shown, and the cutoff is at peak force or the end of the dynamic phase of stretches. The inset shows a photograph of the hindlimbs of an animal with SCl, showing signs of spastic patterns. Stim, Stimulation.

was passed through a $10 \mathrm{M} \Omega$ resistor, then connected to a stainless steel plate electrode (width, $5 \mathrm{~mm}$; length, $9 \mathrm{~mm}$ ) that was placed under the sciatic nerve proximal to the anode electrode. These electrodes were also covered by a layer of wick ( $1 \mathrm{~mm}$ thick; soaked with $0.9 \%$ saline), which was glued to the stainless steel plate. Care was taken to prevent contact among test-stimulating, recording, and DC electrodes. If needed, a small amount of petroleum jelly/silicone oil mixture was used to prevent contact between electrodes. Note that this design allowed for reversal of polarity by switching the polarity of the current source. An ampere meter was connected between $\mathrm{a} 2$ and $\mathrm{c} 2$ branches to monitor the current value. Sciatic nerve electrodes were electrically isolated from the rest of the body by a layer of silicone rubber. This design was devised to solve the chal- 
lenge of delivering relatively higher current values to the spinal cord $(0.4-1 \mathrm{~mA})$ to affect spinal neurons and subthreshold current values $(1-5 \mu \mathrm{A})$ to the sciatic nerve. In exploratory experiments, this design allowed $5 \mu \mathrm{A}$ to pass through the sciatic nerve circuit when $1 \mathrm{~mA}$ passed through the spinal column-abdominal skin circuit. The rationale for placing two electrodes with opposite charges on the sciatic nerve was to reduce the potential damaging effects of monopolar stimulation (Merrill et al., 2005). Given the size of the sciatic nerve (width, $1 \mathrm{~mm}$ ) and the electrode plate (width, $5 \mathrm{~mm}$ ), the exposed nerve area would be $5 \mathrm{~mm}^{2}$. Thus, a current of $5 \mu \mathrm{A}$ applied to the nerve would generate a current density of $1 \mathrm{~A} / \mathrm{m}^{2}$. In all experiments, DCS parameters were $0.8 \mathrm{~mA}$ at the spinal cord and $4.5 \mu \mathrm{A}$ at the sciatic nerve. The duration of DCS was the time of the stretch period: $6 \mathrm{~s}$ in transit stretches and $30 \mathrm{~s}$ in steady-state stretches.

Stretch apparatus. This device was constructed to produce linear motion, which was suitable for inducing muscle stretch in the current setup. It consisted of a high-velocity needle roller-bearing carriage and guide rail. The carriage worked like a trigger with a side handle to lock it along the rail. One side of the carriage was connected to the base by rubber bands, and the pulling velocity was adjusted by changing the number of bands. The other side of the carriage was firmly connected to a force transducer and inertial motion unit (IMU), as shown in Figure 1A. The velocity was measured using a displacement sensor (Measurand) attached to the carriage and video-tracking software. Two rubber bands gave a lengthening velocity of $209 \mathrm{~mm} / \mathrm{s}$ (low velocity), and four bands gave a lengthening velocity of $475 \mathrm{~mm} / \mathrm{s}$ (high velocity). Testing demonstrated that this system was reliable in producing consistent movement velocities of 209 and $475 \mathrm{~mm} / \mathrm{s}$. Moreover, velocity and tracking were measured during each test.

Justification of parameters. TS muscle was stretched $3.5 \mathrm{~mm}$, based on actual TS stretch distance measured in intact animals. Specifically, in intact anesthetized animals, while the knee was flexed, the stretch distance of TS muscle was measured between insertion (ankle) and origin (knee) during full ankle range of motion $\left(0-165^{\circ}\right)$ from full planterflexion to full dorsiflexion. This was also confirmed by cutting the TS tendon, which had a stretch capacity of $3.5 \mathrm{~mm}$. The velocity of the stretches was based on the velocity of the cortically evoked dorsiflexion movement (Ahmed, 2013b), which was between $200 \mathrm{~mm} / \mathrm{s}$ (low velocity) and 500 $\mathrm{mm} / \mathrm{s}$ (high velocity). Therefore, the stretch system was adjusted to produce lengthening movements at the low $(209 \mathrm{~mm} / \mathrm{s})$ and high velocities $(475 \mathrm{~mm} / \mathrm{s})$.

\section{Experimental design}

Experiment 1 (transit stretch responses). To avoid complications, transit stretch responses were tested in separate group of animals (control, $n=$ 5; spinal cord injury, $n=7$ ). Three stretch responses at low and high velocities were elicited at baseline with $1 \mathrm{~min}$ interstretch intervals, followed by either spinal-to-sciatic or sciatic-to-spinal DCS. Spinalabdominal and sciatic-only DCS were tested in different groups of animals, and were found to have no effects on stretch responses. Stretchinduced nerve discharges were recorded, and their frequency and spike histograms were analyzed. Muscle tensions produced by different stretch velocities were recorded and compared. Slope $(\mathrm{g} / \mathrm{s})$, defined as the slope of a regression (least-squares) line, fitted to the data during the rising phase of the reflex force from 5 to $50 \%$ of the peak force, was compared between treatments. In addition, maximal and threshold forces were measured. Threshold force was defined as the force at which nerve discharge began.

Experiment 2 (steady muscle tension). Animals (control, $n=5$; spinal cord injury, $n=9$ ) were used to test the effects of spinal-to-sciatic or sciatic-to-spinal DCS on steady muscle tension. TS muscle was stretched to produce $2 \mathrm{~g}$ of force, and baseline recordings were collected for $3 \mathrm{~min}$. Next, one of the stimulation procedures (spinal-to-sciatic or sciatic-tospinal DCS) was applied for $30 \mathrm{~s}$. After current was turned off, muscle tension was measured for $3 \mathrm{~min}$. The other DCS procedure was then applied for $30 \mathrm{~s}$, and muscle tension was measured for another $3 \mathrm{~min}$. The order of current application was pseudorandom. Data were normalized to baseline. Moreover, nerve discharges were averaged over $30 \mathrm{~s}$ periods and analyzed using spike histograms (frequency of discriminated spikes).
Experiment 3 (Hoffmann reflex, sciatic nerve excitability, and cortically evoked potentials). To investigate the effects of DCS on cortically evoked potentials, a craniotomy was made over the contralateral primary motor cortex (M1; $1 \mathrm{~mm}$ posterior to bregma and $1 \mathrm{~mm}$ lateral to midline) without breaching the dura. A recording electrode (resistance, 1-2 M $\Omega$ ) was inserted into the tibial nerve. The cortex was stimulated with a monopolar electrode (tip, $150 \mu \mathrm{m}$ ), and an active electrode was situated on M1. An alligator clip attached to a flap of scalp skin on the frontal aspect of the skull served as the reference electrode. In addition, to investigate the effects of DCS on Hoffmann reflex (H-reflex), a concentric bipolar stimulating electrode (tip, $250 \mu \mathrm{m}$ ) was placed on the sciatic nerve in the pelvis region. In the same group of animals $(n=5)$, H-reflex and cortically evoked potentials were recorded before, during, and after spinal-tosciatic or sciatic-to-spinal DCS. Since no long-term effects were observed after these brief test periods (10 s), data recorded after DCS application were not included in the analysis.

A separate group $(n=6)$ of animals was used to test the local effects of DCS on the sciatic nerve. In proximal test stimulation, the test stimulus electrode was placed over the cephalic side of the sciatic nerve, and the recording electrode was placed on the distal side near the muscle. Test stimulation was also performed at the nerve segment located between the two DCS electrodes. In these experiments, baseline nerve compound action potentials were adjusted to $\sim 50 \%$ of maximal compound action potential. A train of five nerve compound action potentials (at $0.5 \mathrm{~Hz}$ ) was evoked before (baseline), during, and after spinal-to-sciatic or sciatic-to-spinal DCS. Testing was performed in a pseudorandom order with $2 \mathrm{~min}$ intervals between tests. To examine the nerve for any damage caused by DCS, nerve compound action potentials and concurrent muscle twitches were followed for $20 \mathrm{~min}$ after termination of the DCS procedure in four experiments. Trains of five pulses at $0.5 \mathrm{~Hz}$ were applied at 5 min intervals, and average potentials and twitches were compared with baseline values.

To test the excitability of the proximal sciatic nerve segment, the sciatic nerve was exposed further toward the pelvis region in a group of animals $(n=5)$. A recording electrode was placed proximally on the pelvic part of the sciatic nerve, and a test-stimulating electrode was placed caudal to the recording electrode, but rostral to the DC electrodes. A train of five nerve compound action potentials (at $0.5 \mathrm{~Hz}$ ) was antidromically evoked before (baseline), during, and after spinal-tosciatic or sciatic-to-spinal DCS.

All extracellular recordings were passed through a standard head stage, amplified (Neuro Amp EX, ADInstruments), filtered (100 Hz-5 kHz bandpass filter), digitized at $4 \mathrm{kHz}$, and stored in the computer for further processing. A PowerLab data acquisition system and LabChart 7 software (ADInstruments) were used to acquire and analyze the data.

\section{Statistic analysis}

Data in all graphs represent means \pm SEM. The differences between groups over time were assessed using repeated-measures ANOVA (independent variables: time course and stimulation condition; dependent variables: muscle resistance, firing rate of nerve discharges, maximal force, threshold force, nerve compound action potential, H-reflex, and cortically evoked potential) with a Holm-Sidak post hoc correction to test differences across time points. $t$ tests were used to compare control and spinal cord injury groups. Pearson correlations were used to test correlations between stretch velocity and the following variables: threshold force, maximal force, and nerve discharge frequency. Statistical analyses were performed using SigmaPlot (SPSS). Slopes, maximal force, and spike rate were calculated using LabChart software (ADInstruments). The critical level of significance was set at $p<0.05$.

\section{Results}

\section{Effects of DCS on transit stretch responses}

Stretch-induced discharges

To test the effects of DCS on transit stretch-induced discharges, low-velocity stretches were performed over $6 \mathrm{~s}$ during spinal-tosciatic or sciatic-to-spinal DCS, and maximal nerve discharge rates were measured. As shown by ANOVA (Table 1), the main 
Table 1. Effects of DCS on transit stretch responses

\begin{tabular}{|c|c|c|c|c|c|c|c|}
\hline \multirow[b]{2}{*}{ Outcome measures } & \multirow[b]{2}{*}{ Baseline } & \multirow{2}{*}{$\begin{array}{l}\text { During spinal-to- } \\
\text { sciatic DCS }\end{array}$} & \multirow{2}{*}{$\begin{array}{l}\text { During sciatic-to- } \\
\text { spinal DCS }\end{array}$} & \multicolumn{2}{|c|}{ Overall (ANOVA) } & \multicolumn{2}{|l|}{$p$ value (post hoc comparison) } \\
\hline & & & & Fvalue & $p$ value & Baseline vs spinal-to-sciatic DCS & Baseline vs sciatic-to-spinal DCS \\
\hline Nerve discharges in controls (spikes/s) & $21.8 \pm 1.3$ & $10.8 \pm 1.8$ & $46.5 \pm 0.7$ & 137.0 & $<0.001^{*}$ & $<0.002^{*}$ & $<0.002^{*}$ \\
\hline Slope of muscle resistance in controls $(\mathrm{g} / \mathrm{s})$ & $80.2 \pm 16.4$ & $68.9 \pm 25.2$ & $177.9 \pm 30.9$ & 6.3 & $<0.02^{*}$ & $<0.03^{*}$ & $<0.03^{*}$ \\
\hline Slope of muscle resistance in mice with $\mathrm{SCl}(\mathrm{g} / \mathrm{s})$ & $359.4 \pm 0.4$ & $272.3 \pm 8.5$ & $676.5 \pm 89.8$ & 14.1 & $<0.001^{*}$ & $<0.03^{*}$ & $<0.03^{*}$ \\
\hline
\end{tabular}

Values are given as the mean $\pm S D$, unless otherwise stated.

*Significant value.
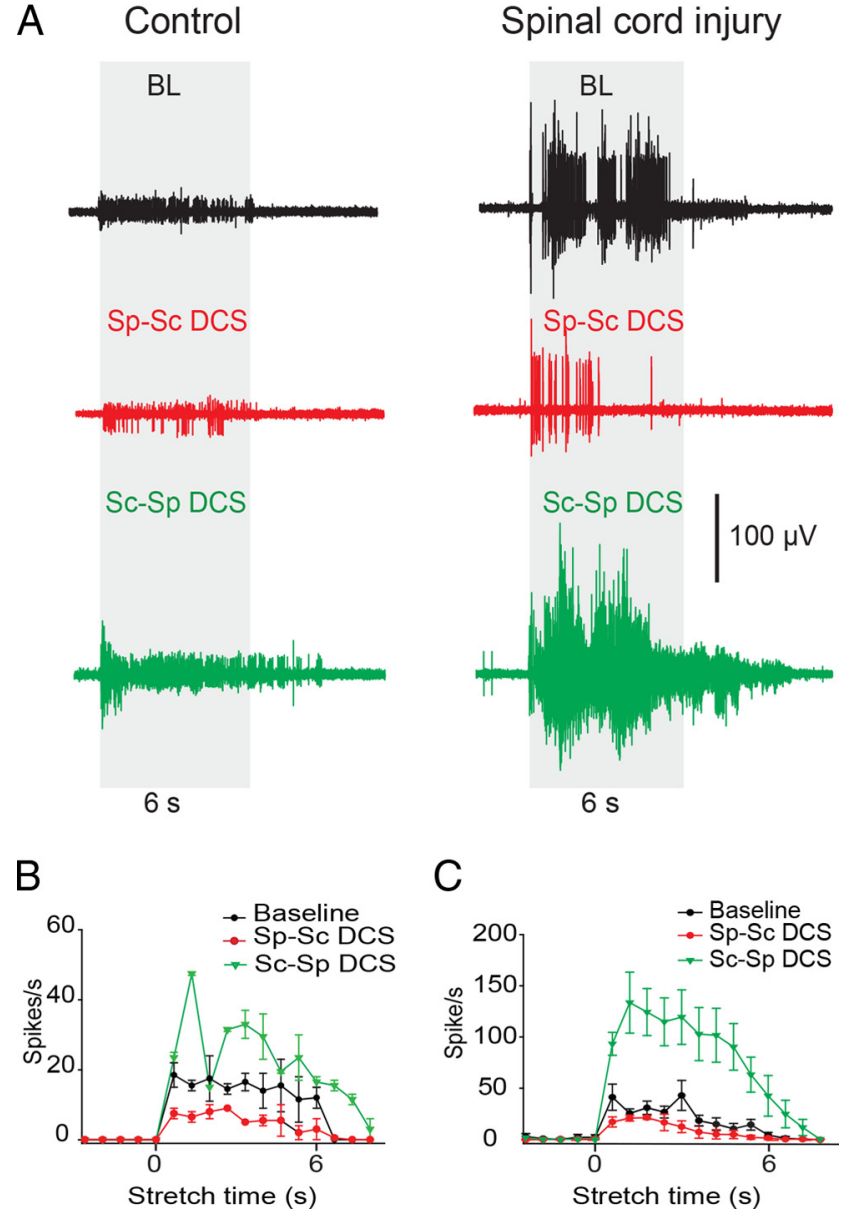

Figure 2. DCS altered slow-velocity, stretch-induced nerve discharges in control and injured animals. $\boldsymbol{A}$, Examples of stretch-induced discharges during the following three conditions: (1) no-DCS [baseline (BL)], top (black); (2) spinal-to-sciatic (Sp-Sc) DCS, middle (red); and (3) sciatic-to-spinal (Sc-Sp) DCS, bottom (green). B, Summary plot showing stretch-induced discharges in control animals. $\boldsymbol{C}$, Summary plot showing stretch-induced discharges in animals with SCI. Data are shown as the mean \pm SEM.

effect of stimulation on nerve discharges was significant in control animals. As shown in Figure $2 A$, in control animals, compared with baseline, the maximal firing rate was decreased by spinal-to-sciatic DCS, and increased by sciatic-to-spinal DCS (Table 1 ). Figure $2 B$ shows the changes in stretch-induced nerve discharges during the entire $6 \mathrm{~s}$ transit stretch period. Spinal-tosciatic DCS (Fig. 2B, red) reduced stretch responses during the dynamic phase and discharges during the static phase of the stretch period. Sciatic-to-spinal DCS (Fig. $2 B$, green) increased the rate of nerve discharges during both dynamic and static phases of the stretch period, and elongated the after-stretch response. There were no poststimulation changes in stretchinduced discharges.
As shown by ANOVA (Table 1), the main effect of stimulation on nerve discharges was significant in animals with spinal cord injury. In Figure $2 A$, in animals with spinal cord injury, compared with baseline, maximal firing rate of stretch-induced discharges was decreased by spinal-to-sciatic DCS and increased by sciatic-to-spinal DCS (Table 1). Figure $2 C$ shows the changes in nerve discharges during the entire $6 \mathrm{~s}$ transit stretch period. Spinal-to-sciatic DCS reduced nerve discharges during the dynamic phase, reduced and shortened discharges during static phase, and inhibited the after-stretch discharges. Sciatic-tospinal DCS increased nerve discharges during the dynamic and static phases, and enhanced and prolonged the after-stretch discharges (Table 1). In all conditions, stretch-induced discharges returned to baseline values after stimulation ended. In response to low-velocity stretches, animals with SCI injury had significantly higher discharge rates at baseline compared with control animals ( $t$ test, $p<0.02$ ).

\section{Stretch-induced muscle resistance}

As shown by ANOVA (Table 1), the main effect of stimulation on muscle resistance was significant in control animals. In Figure 3, $A$ and $B$, in control animals compared with baseline, the slope of muscle resistance at low velocity was decreased by spinal-tosciatic DCS, and increased by sciatic-to-spinal DCS (Table 1). As shown by ANOVA (Table 1), the main effect of stimulation on muscle resistance was significant in animals with SCI. In Figure 3, $C$ and $D$, in animals with SCI compared with baseline, the slope of muscle resistance was decreased by spinal-to-sciatic DCS and increased by sciatic-to-spinal DCS (Table 1). There were no persistent effects after stimulation ended. Animals with SCI had higher resistances at baseline compared with controls (one-way ANOVA, $F=7.9, p<0.001$ ), indicating that SCI caused an exaggeration in reflex force. At equivalent current intensities, neither spinal-abdominal nor sciatic-only DCS caused changes in transit stretch responses (data not shown).

\section{Effects of DCS on velocity-sensitive parameters}

Next, stretches were performed in animals with spinal cord injury at low velocity $(209 \mathrm{~mm} / \mathrm{s})$ and high velocity $(475 \mathrm{~mm} / \mathrm{s})$. The following three outcome measures were analyzed: (1) rate of nerve discharges during the dynamic period of the stretches; (2) maximal force, defined as the peak force at the end of the dynamic period; and (3) threshold force, defined as the force at which nerve discharges begin.

In Figure 4, at baseline, stretch velocity was positively correlated with the rate of nerve discharges and maximal force (Pearson correlation, $p<0.05)$, but not threshold force $(p>0.05)$. During spinal-to-sciatic DCS, stretch velocity was not correlated with rate of nerve discharges or maximal force (Pearson correlation, $p>0.05)$, but was negatively correlated with stretch velocity $(p<0.05)$. During sciatic-to-spinal DCS, velocity was not correlated with rate of nerve discharges, maximal force, or threshold 

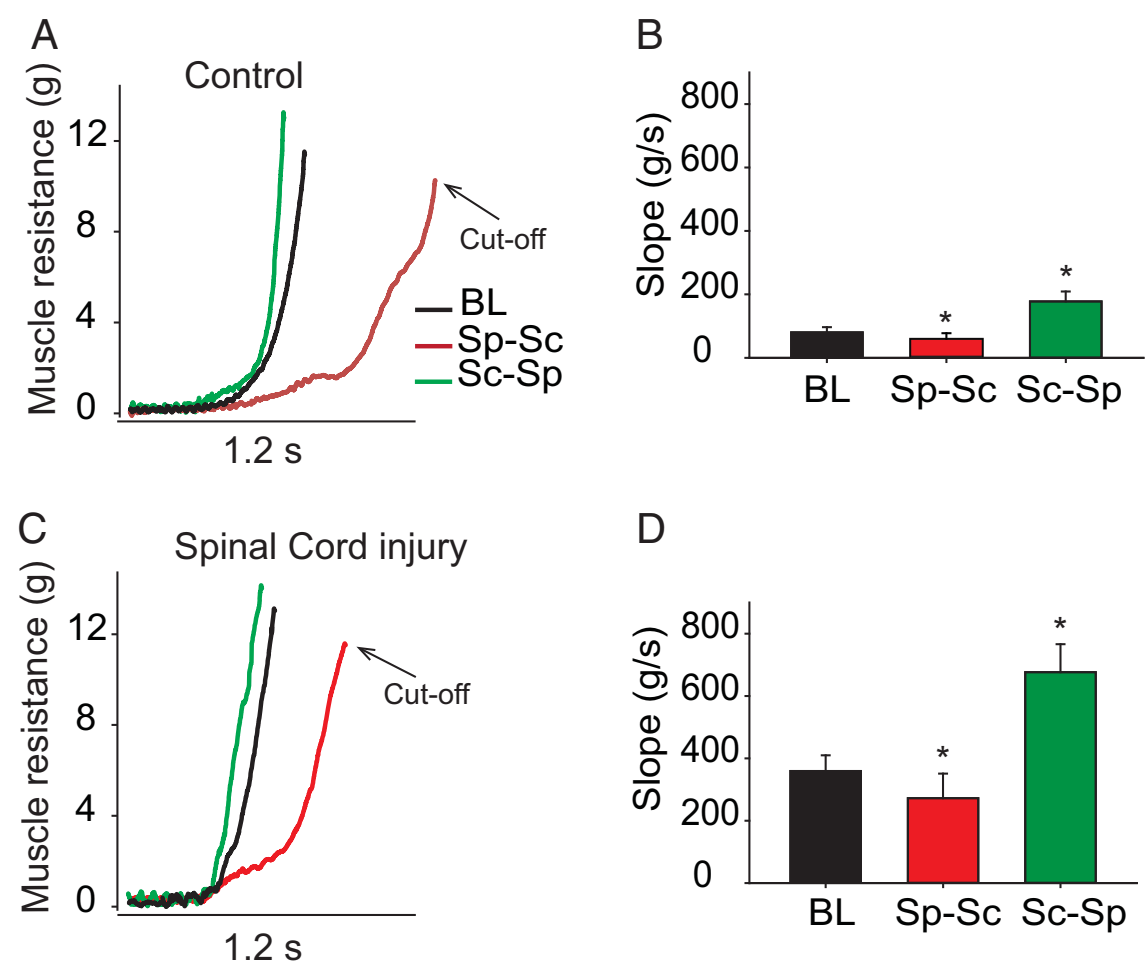

Figure 3. DCS altered the slope of muscle resistance during the dynamic phase of stretch. $\boldsymbol{A}$, Traces of muscle resistance recorded during low-velocity stretches in control animals. Cutoff ends at peak force. $B$, Summary plot showing that the slope of muscle resistance in control animals was reduced by spinal-to-sciatic (Sp-Sc) DCS and increased by sciatic-to-spinal (Sc-Sp) DCS. C, Traces of muscle resistance recorded during low-velocity stretches in animals with SCI. D, Summary plot showing that the slope of muscle resistance in animals with SCI was reduced by spinal-to-sciatic DCS and increased by sciatic-t0-spinal DCS. * $p<0.05$. Data are shown as the mean \pm SEM. force (Pearson correlation, $p>0.05$ ) These findings indicate that stretch responses at baseline are velocity dependent, which is an important criterion of spasticity. Conversely, DCS effects were velocity independent, suggesting that DCS has greater effects on the tonic component of the stretch reflex. One exception was spinal-to-sciatic DCS, which induced a negative correlation of stretch velocity with threshold force.

Further analysis revealed that at low and high velocities DCS had main effects on maximal force (repeated-measures ANOVA: $F=5.8, p<0.01$; and $F=10.8$, $p<0.002$, respectively), threshold force (repeated-measures ANOVA: $F=7.4$, $p<0.01$; and $F=13.5, p<0.001$, respectively), and rate of nerve discharges (repeated-measures ANOVA: $F=25.7$, $p<0.001$; and $F=15.6, p<0.001$, respectively). In Figure 4, compared with baseline, maximal force produced at low and high velocities was reduced during spinal-to-sciatic DCS and increased during sciatic-to-spinal DCS (Holm-Sidak method, $p$ values $<0.05)$. Threshold force at low and high velocities was increased by spinal-to-sciatic DCS (Holm-Sidak method, $p<0.02$ and $p<0.001$, respectively), but was not affected by sciatic-tospinal DCS ( $p$ values $>0.05)$. Nerve discharges at high and low velocities were reduced by spinal-to-sciatic DCS (Holm-Sidak method, $p<$ 0.001 and $p<0.03$, respectively), but were increased by sciaticto-spinal DCS ( $p<0.02$ and $p<0.007$, respectively). In all stimulation conditions, there were no persistent effects after the stimulation ended.
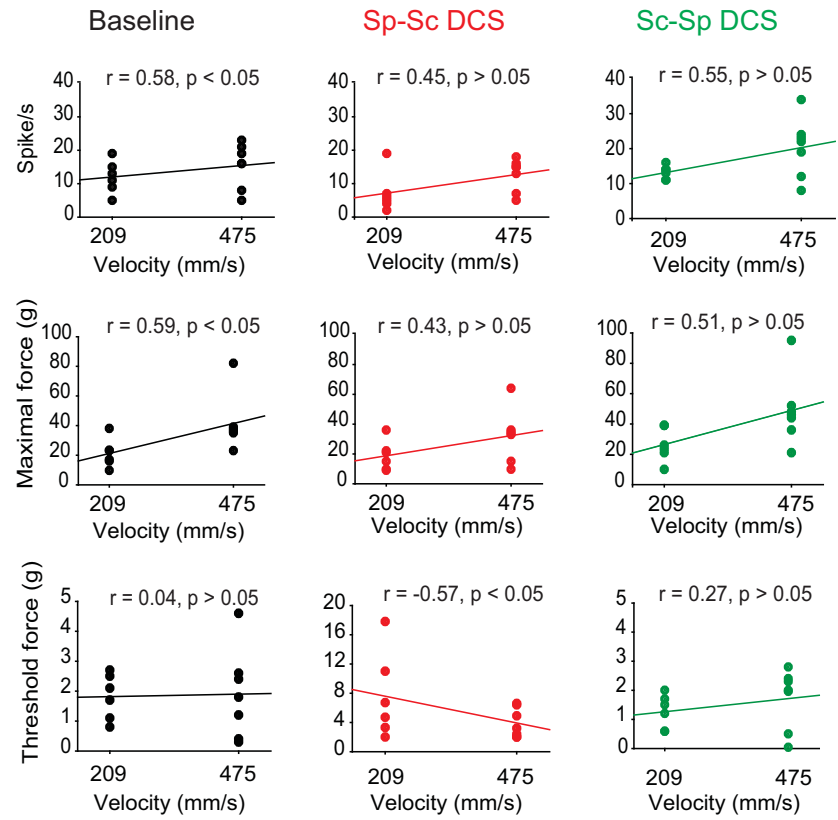

Figure 4. Scatter plots showing the effects of lengthening velocity and DCS on maximal force, firing rate, and threshold force in mice with SCI. At baseline (left panels, black trace), both maximal force and firing rate were velocity dependent; however, threshold force was velocity independent. During spinal-to-sciatic (Sp-Sc) DCS (middle panels, red trace), both maximal force and firing rate were velocity independent; however, threshold force was velocity dependent. During sciatic-to-spinal (SC-Sp) DCS (right panels, green trace), maximal force, firing rate, and threshold force were velocity independent.

\section{Effects of DCS on steady-state muscle tension and nerve discharges}

To test the effects of DCS on steady muscle tension, muscles were put under a relatively moderate constant tension of $2 g$ (average threshold force). In control animals, repeated-measures ANOVA revealed the main effect of DCS on muscle tension and nerve discharges (Table 2). Compared with baseline, muscle tension was decreased by spinal-to-sciatic DCS and increased by sciaticto-spinal DCS. Similarly, compared with baseline, the nerve discharge rate was decreased by spinal-to-sciatic DCS and increased by sciatic-to-spinal DCS (Table 2). There were no persistent effects after stimulation ended.

In Figure 5, in animals with spinal cord injury, repeatedmeasures ANOVA revealed significant general effects of DCS on muscle tension and nerve discharges (Table 2). Compared with baseline, muscle tension was decreased by spinal-to-sciatic DCS and increased by sciatic-to-spinal DCS. Similarly, compared with baseline, the nerve discharge rate was decreased during spinal-tosciatic DCS and increased during sciatic-to-spinal DCS (Table 2). There were no persistent effects after stimulation ended. At equivalent current intensities, neither spinal-abdominal nor sciatic-only DCS caused changes in steady muscle tension (data not shown). 
Table 2. Effects of DCS on steady-state muscle tension and nerve discharges

\begin{tabular}{|c|c|c|c|c|c|c|c|}
\hline \multirow[b]{2}{*}{ Outcome measures } & \multirow[b]{2}{*}{ Baseline } & \multirow{2}{*}{$\begin{array}{l}\text { During spinal-to- } \\
\text { sciatic DCS }\end{array}$} & \multirow{2}{*}{$\begin{array}{l}\text { During sciatic-to- } \\
\text { spinal DCS }\end{array}$} & \multicolumn{2}{|c|}{ Overall (ANOVA) } & \multicolumn{2}{|l|}{$p$ value ( post hoc comparison) } \\
\hline & & & & Fvalue & $p$ value & Baseline versus spinal-to-sciatic DCS & Baseline versus sciatic-to-spinal DCS \\
\hline Nerve discharges in controls (spikes/s) & $5.5 \pm 0.7$ & $3.1 \pm 0.6$ & $34.6 \pm 2.3$ & 134.0 & $<0.001^{*}$ & $<0.01^{*}$ & $<0.01^{*}$ \\
\hline Nerve discharges in mice with SCI (spikes/s) & $14.9 \pm 3.6$ & $3.5 \pm 2.2$ & $90.0 \pm 2.9$ & 47.4 & $<0.001^{*}$ & $<0.001^{*}$ & $<0.001^{*}$ \\
\hline Muscle resistance in controls $(g$ ) & $2.0 \pm 0.0$ & $1.8 \pm 0.1$ & $2.4 \pm 0.1$ & 58.6 & $<0.001^{*}$ & $<0.009^{*}$ & $<0.001^{*}$ \\
\hline Muscle resistance in mice with SCI ( $g$ ) & $2.0 \pm 0.0$ & $1.7 \pm 0.1$ & $2.5 \pm 0.1$ & 17.1 & $<0.001^{*}$ & $<0.001^{*}$ & $<0.001^{*}$ \\
\hline
\end{tabular}

Values are given as the mean \pm SEM, unless otherwise stated.

*Significant value.

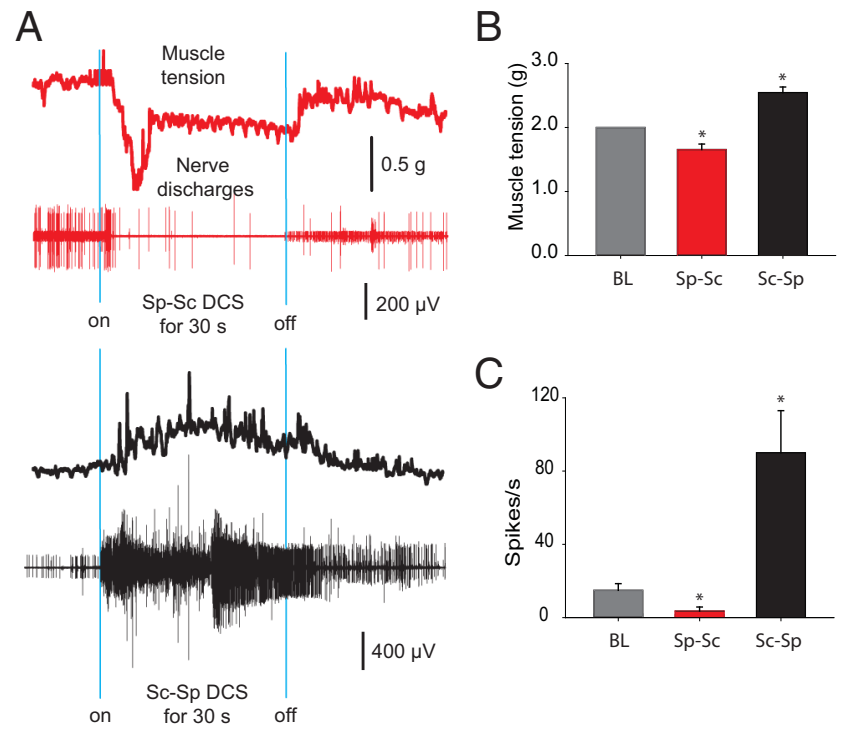

Figure 5. Effects of DCS on steady-state muscle tension. TS muscle was slightly stretched (2 $g$ tension). $A$, Representative muscle force traces and concurrent nerve discharges during DCS. Top (red trace), Spinal-to-sciatic (Sp-Sc) DCS reduced muscle tension and nerve discharges. Bottom (black trace), Sciatic-to-spinal (SC-Sp) DCS increased muscle tension and nerve discharges. $B$, Summary plot showing that muscle tension was decreased by spinal-to-sciatic DCS, but was increased by sciatic-to-spinal DCS. ${ }^{*} p<0.05$. C, Summary plot showing that nerve discharges were decreased by spinal-to-sciatic DCS, but were increased by sciatic-to-spinal DCS. ${ }^{*} p<0.05$. Data are shown as the mean \pm SEM.

Effects of DCS on H-reflex, sciatic nerve excitability, and cortically evoked potentials in animals with spinal cord injury The DCS configuration used in the present study was different from the configuration used in our previous studies (Ahmed, 2011, 2013a,b; Ahmed and Wieraszko, 2012). Therefore, replicating these parametric studies was crucial. In addition, testing local excitability changes in the stimulated nerve segment was important for the following two reasons: (1) to verify that there was no current-induced block; and (2) to localize the site of DCS effects by comparing local nerve changes to changes in cortically evoked potentials.

Repeated-measures ANOVA showed significant main effects of DCS on H-reflex $(F=16.5, p<0.001)$. Compared with baseline $[79.0 \pm 3.5 \%$ of muscle response ( $\mathrm{M}$ wave) $], \mathrm{H}$-reflex was reduced by spinal-to-sciatic DCS and increased by sciatic-tospinal DCS $(62.3 \pm 4.3 \%$ and $94.7 \pm 10.1 \%$ of $M$ wave, respectively; $n=7 ; p<0.02$, Holm-Sidak method).

Six experiments were performed to test the effects of DCS on nerve excitability. The sciatic nerve was test stimulated proximal to the electrodes on the sciatic nerve and between the two electrodes. Nerve compound action potentials were recorded distally, as shown in Figure 6 (top diagrams). Test stimulation was adjusted to produce $\sim 50 \%$ of the maximal nerve compound action potential. Compared with baseline $(1318.2 \pm 51.3 \mu \mathrm{V})$, nerve compound action potential was decreased during spinal-tosciatic DCS when test stimulation was applied proximal to the electrodes ( $1140.6 \pm 50.9 \mu \mathrm{V} ; p<0.001$, paired $t$ test), as shown in Figure 6A. However, compared with baseline (1008.4 \pm 45.8 $\mu \mathrm{V})$, nerve compound action potential was increased during spinal-to-sciatic DCS when test stimulation was applied between the two DCS electrodes $(1553.0 \pm 24.8 \mu \mathrm{V} ; p<0.001$, paired $t$ test), as shown in Figure 6B. Compared with baseline (1103.0 \pm $29.4 \mu \mathrm{V})$, nerve compound action potential was increased by sciatic-to-spinal DCS with test stimulation proximal to the electrodes $(1310.1 \pm 105.2 \mu \mathrm{V} ; p<0.001$, paired $t$ test $)$, as shown in Figure $6 C$. However, relative to baseline $(1538.2 \pm 3.6 \mu \mathrm{V})$, the nerve compound action potential was decreased during sciaticto-spinal DCS with test stimulation between the two electrodes (1376.4 $\pm 27.4 \mu \mathrm{V} ; p<0.02$, paired $t$ test), as shown in Figure $6 D$. During spinal-to-sciatic DCS, nerve compound action potential increased when test stimulation was applied at the nerve segment facing the cathode electrode and decreased when test stimulation was applied at the nerve segment facing the anode electrode. In all of these experiments, nerve compound action potential reverted to baseline values following the cessation of DCS. In four experiments, nerve compound action potentials and concurrent muscle twitches were assessed for 20 min following the end of the DCS procedure. There was no sign of loss of excitability (Agnew et al., 1989) or nerve-muscle coupling by DCS (Fig. 6E).

To investigate the effects of DCS on the excitability of the proximal segment of the sciatic nerve (between the spinal and sciatic DC electrodes), the sciatic nerve in the pelvic region was exposed and a recording electrode was placed over it $\sim 2 \mathrm{~cm}$ rostral from the sciatic nerve DC electrode. The test-stimulating electrode was also placed proximal to the sciatic nerve DC electrode (Fig. 7A). One-way repeated-measures ANOVA showed a significant effect during spinal-to-sciatic DCS $(F=9.1, p=$ 0.009 ). The size of the nerve compound action potential was significantly reduced (baseline, $778.6 \pm 30.4 \mu \mathrm{V}$; spinal-tosciatic DCS, $588.0 \pm 64.2 \mu \mathrm{V} ; p<0.003$, Holm-Sidak method) but returned to baseline levels after spinal-to-sciatic DCS was turned off $(726.8 \pm 42.5 \mu \mathrm{V} ; p=0.3$, Holm-Sidak method; Fig. $7 B, C)$. One-way repeated-measures ANOVA showed a significant effect during sciatic-to-spinal DCS $(F=7.3, p<0.01)$. The compound action potential was significantly increased during sciatic-to-spinal DCS (baseline, $718.6 \pm 70.4 \mu \mathrm{V}$; sciatic-tospinal DCS, $1067.8 \pm 65.8 \mu \mathrm{V} ; p=0.008$, Holm-Sidak method) but returned to baseline levels after DCS was turned off (764.6 \pm $75.4 \mu \mathrm{V} ; p=0.7$, Holm-Sidak method; Fig. $7 B, C)$. Recordings were also performed in multiple sites along the proximal sciatic nerve segment, yielding identical results. These findings indicate that excitability changes span the entire proximal sciatic nerve segment, extending from the spinal cord to the sciatic nerve DC electrode.

Because the DCS stimulation arrangement used in the present study is different from the previous arrangement (Ahmed, 
A

B

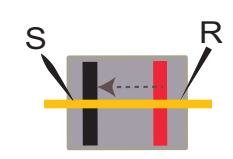

BL $\neg$
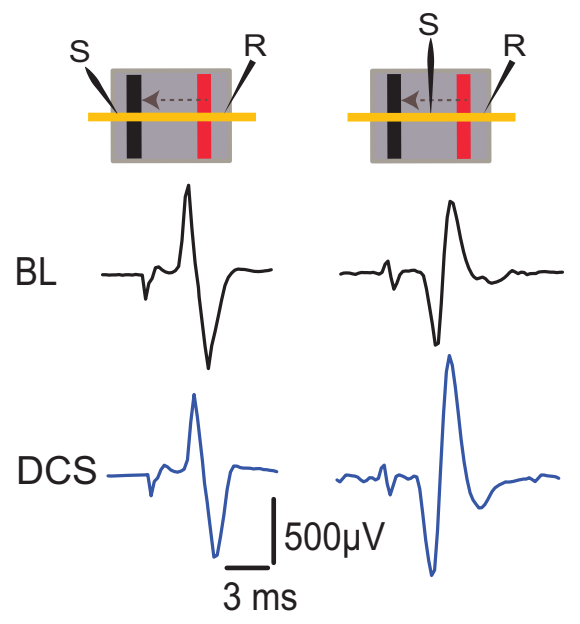

C
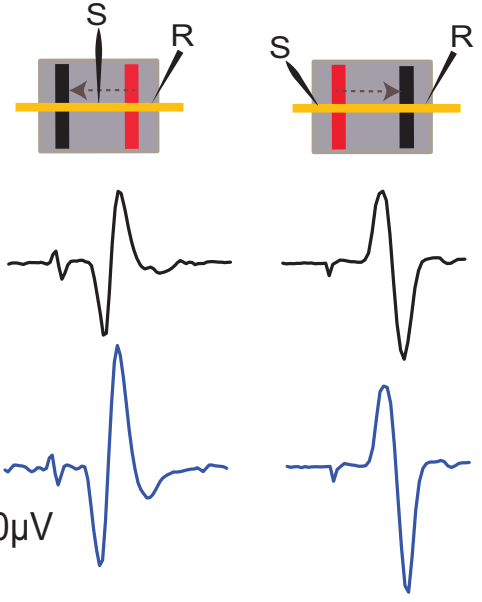

\section{$E$}

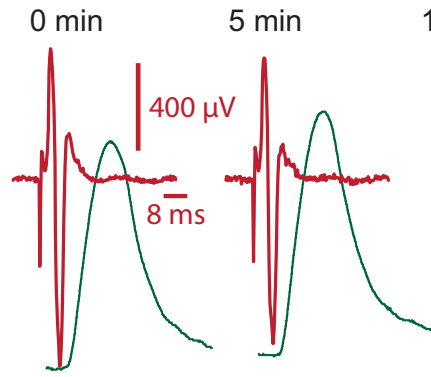

$10 \min$
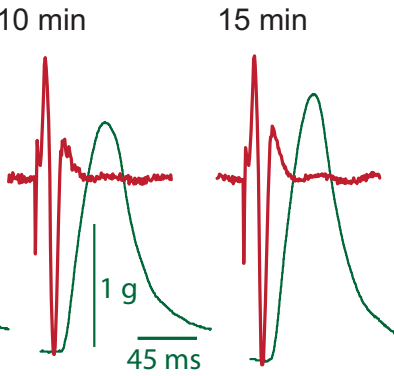

Figure 6. Effects of DCS on local nerve excitability. $A, D C S$-induced changes at the nerve. Insets at the top of the figure show the arrangement of stimulating $(S)$ and recording $(\mathrm{R})$ electrodes, and $\mathrm{DC}$ electrodes (cathode, black; anode, red) on the sciatic nerve (yellow horizontal line). Arrows indicate the direction of current. Note that the recording electrode is located caudal to the body, and the stimulating electrode rostral to the body. The dark background represents the silicone rubber that isolates the nerve and electrodes from the rest of the body. The lower panels show traces of nerve compound action potential recorded during baseline $(B L)$, and during spinal-to-sciatic DCS. B, Spinal-to-sciatic DCS increased nerve compound action potential evoked by test stimulation between the two DCS electrodes. C, Sciatic-to-spinal DCS increased nerve compound action potential evoked by distal test stimulation. $D$, Spinal-to-sciatic DCS reduced nerve compound action potential evoked by test stimulation between the two sciatic DCS electrodes. $E$, Examples of nerve compound action potentials (red) and concurrent muscle twitches (green) recorded after DCS $(0-20 \mathrm{~min})$. This shows no damaging effect of DCS protocols used in the present study.

2013a), it is important to assess the changes in cortically evoked potentials that accompanied the observed changes in muscle tone. Compared with baseline $(277.8 \pm 31.1 \mu \mathrm{V})$, cortically evoked potentials were reduced by spinal-to-sciatic DCS $(198.4 \pm 41.6 \mu \mathrm{V} ; p<0.03$, paired $t$ test; $n=5)$. Conversely, compared with baseline $(220.6 \pm 17.3 \mu \mathrm{V})$, potentials were increased by sciatic-to-spinal DCS $(335.2 \pm 29.2 \mu \mathrm{V} ; p<0.01$, paired $t$ test; $n=5)$. Overall, these results suggest that DCS affects muscle tone by modulating spinal cord excitability.

\section{Discussion}

The present study tested the effects of trans-spinal sciatic-tospinal or spinal-to sciatic DCS on physiological and pathological muscle tone. The two lengthening velocities used were within the range that can be produced by cortically evoked dorsiflexion in mice (Ahmed, 2013b). In addition, the TS muscle was stretched within its normal range of motion. Muscle stretch evoked a velocity-dependent increase in muscle resistance in mice with chronic contusive spinal cord injury, supporting previous studies (Thompson et al., 1996; Taylor et al., 1997; Bose et al., 2002; Marsala et al., 2005). These animals had also demonstrated clear signs of spasticity, such as spasms, clonus, and digit fanning (Fig.

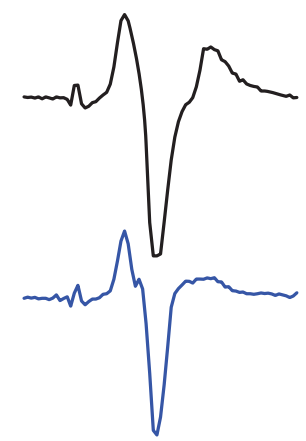

$20 \min$

1). In addition, stretch-induced nerve discharges were higher in animals with spinal cord injury.

The DCS setup used in the present study uses two current paths. The first path involved current flow between the spinal cord and abdominal skin. This current path was previously demonstrated to cause inhibition with an anodal spinal electrode and cathodal abdominal electrode or excitation when current flowed in the opposite direction (Marsan et al., 1951; Ahmed, 2011; Ahmed and Wieraszko, 2012). Relatively higher current intensity is needed in the spinal-abdominal current path to have consistent effects on spinal motor neurons and interneurons. The need for higher current intensities at the spinal cord might be due to the larger conductive volume, and the relatively greater distance between the spinal cord and the electrode. The second path involved current flow between the dorsal surface of the spinal cord and sciatic nerve. That is, an anodal spinal electrode and cathodal motor nerve electrode depresses activation of motoneurons; the opposite arrangement facilitates activation of motoneurons. The current required to activate this path is very low, ranging from 2 to $5 \mu \mathrm{A}$. In exploratory experiments, higher intensities induced local firing of the sciatic nerve due to its small diameter, which caused high local current density and could lead to anodal block. Thus, the present study combined the two current paths. It should be noted that spinal-to-sciatic DCS was predicted to depress Ia afferent inputs. Eccles et al. (1962) found that an anode on the dorsum of the spinal cord reduced EPSP size at the synapses between primary afferents and motoneurons. This hypothesis was also supported by the finding that responses to sensory stimulation were reduced by currents flowing from spinal cord to peripheral axons (Barron and Matthews, 1938).

Spinal-to-sciatic DCS reduced both stretch-induced nerve discharges and muscle resistance, and sciatic-to-spinal DCS produced opposite effects. Threshold force, at which nerve discharges began, was not velocity dependent at baseline or during sciatic-to-spinal DCS, but was velocity dependent during spinalto-sciatic DCS. The change in threshold force illustrates alteration in the readiness of the muscle spindle, which indicates possible effects of DCS on gamma motor neurons. Reducing the excitability of dynamic gamma motor neurons would reduce the dynamic sensitivity of primary sensory endings (Matthews, 1972), which would, in turn, increase the threshold force. Altogether, the velocity dependence of threshold force observed during spinal-to-sciatic DCS appears to indicate a reduced excitability of dynamic gamma motor neurons that is overcome by higher-velocity stretches. The maximal force and discharge rate of concurrent nerve activity were velocity dependent during baseline conditions, but not during spinal-to-sciatic or sciatic-to- 
spinal DCS (Fig. 4). This suggests that DCS affects spinal motoneurons by increasing or decreasing excitability drive from spinal interneurons that are not involved in the stretch reflex (Ahmed, 2013b), akin to the effect of brainstem on spinal motoneuron pools (Takakusaki, 2008). However, H-reflex was attenuated by spinal-to-sciatic DCS and increased by sciatic-to-spinal DCS, suggesting that modulation of transmission from group Ia afferents to motoneurons by DCS may be involved in reducing stretch responses. Overall, these results indicate that DCS affects the background activity of spinal motoneuron pools, as well as specific pathways (e.g., velocity-dependent stretch reflex), but its effects on background activity minimize its effects on specific pathways.

Steady muscle tension and concurrent nerve activity were reduced by spinal-tosciatic DCS and increased by sciatic-tospinal DCS. As noted above, stretching a muscle causes dynamic responses during the acceleration phase of the stretch and static responses during the steady state. The steady-state response is mediated by group II muscle spindle afferents and modulated by static gamma motor neurons (Matthews, 1972). The decrease in muscle tension during spinal-to-sciatic DCS could reflect a change in excitability of static gamma motor neurons or $\alpha$ motoneurons, or effects on upstream spinal interneurons. The increase in muscle tension during sciatic-to-spinal DCS suggests the involvement of opposite mechanisms.

The present results suggest that transspinal spinal-to-sciatic or sciatic-tospinal DCS acts on the spinal cord and proximal sciatic nerve segment. Cortically evoked potentials were decreased by spinal-to-sciatic DCS and increased by sciatic-to-spinal DCS. However, excitability of the distal segment of the sciatic nerve (near the muscle) was increased during spinal-to-sciatic DCS (Fig. 6B) and decreased during sciatic-to-spinal DCS (Fig. 6D). Thus, the changes induced by the current in the distal segment of the sciatic nerve would have an effect on the cortically evoked potential opposite to that actually observed. The observed effect of spinal-to-sciatic or sciatic-tospinal DCS on cortically evoked potential must therefore be produced by its action on the spinal cord/proximal sciatic nerve segment. In support of that conclusion, the excitability of the proximal segment of the sciatic nerve is decreased during spinal-to-sciatic DCS (Figs. $6 A, 7 B, C$ ) and is increased during sciatic-to-spinal DCS (Figs. $6 C, 7 B, C)$. Altogether, these results indicate the following: (1) local changes in the excitability of the distal sciatic nerve segment

A

C
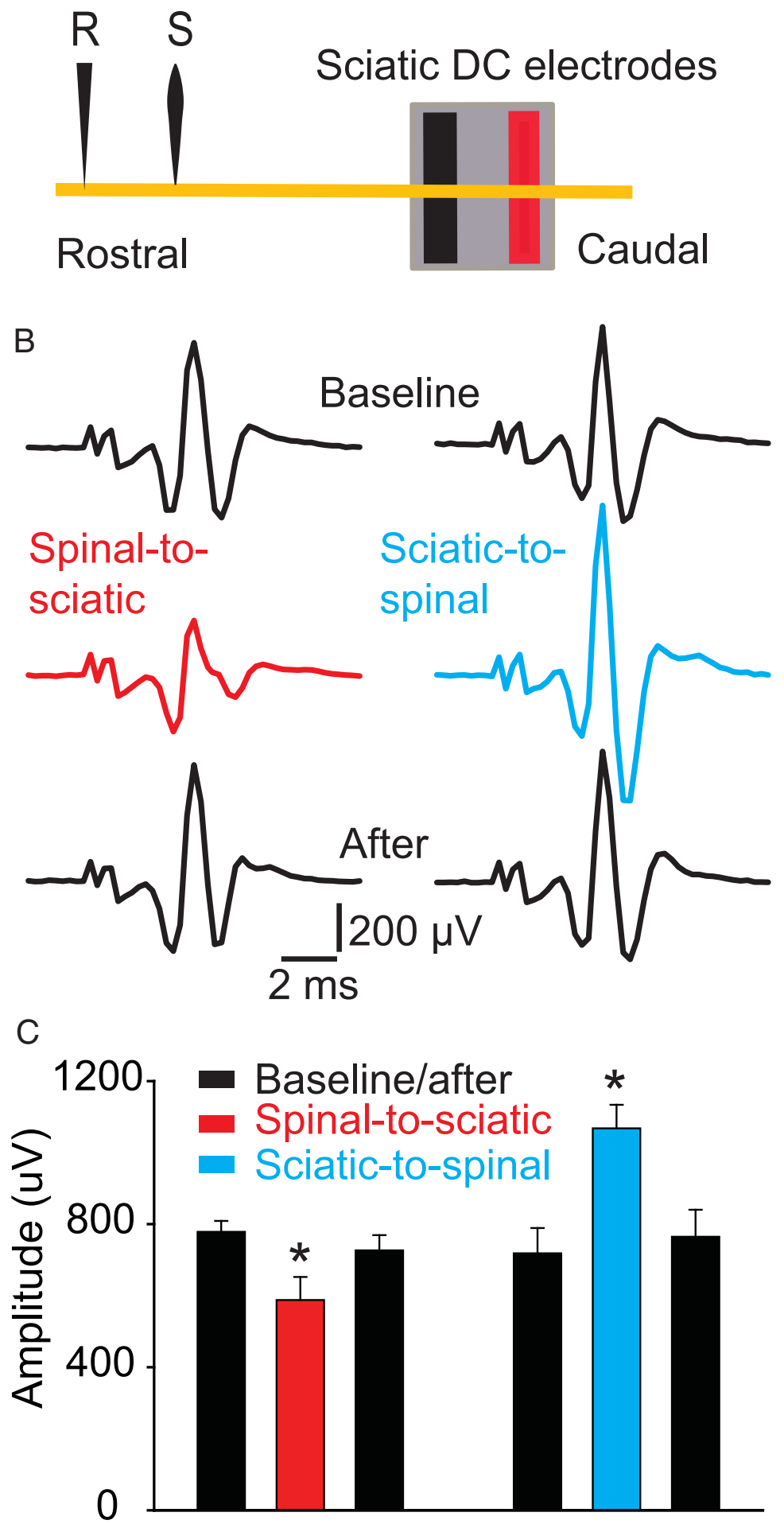

Figure 7. Effects of DCS on the proximal segment of the sciatic nerve. $A$, Schematic diagram showing the experimental setup. The yellow line represents the sciatic nerve. Note that the recording electrode $(R)$ was located $\sim 2 \mathrm{~cm}$ rostral to the sciatic nerve $D C$ electrode. The stimulating electrode $(S)$ was $\sim 7 \mathrm{~mm}$ caudal to the recording electrode. $\boldsymbol{B}$, Examples of nerve compound action potentials recorded antidromically. $\boldsymbol{C}$, Summary plot showing that the nerve compound action potential was reduced during spinal-to-sciatic DCS and increased during sciatic-to-spinal DCS. ${ }^{*} p<0.05$. Data are shown as the mean \pm SEM. are not a factor in the action of trans-spinal DCS; however, (2) excitability changes in the proximal sciatic nerve segment are a critical factor in modulating DCS-induced muscle tone changes. This is also supported by the finding that the application of 
current to only a sciatic nerve circuit or an abdominal circuit had no effect on muscle tone; simultaneous stimulation of both circuits was required to change muscle tone.

The present results are the first demonstration of trans-spinal DCS-induced alterations in muscle tone, and they have obvious clinical applications. Trans-spinal DCS could be applied noninvasively to humans to treat or manage muscle tone abnormalities. Moreover, trans-spinal DCS could be applied through implantable electrodes to manage severe spastic conditions (e.g., spastic bladder). In addition, since spinal-to-sciatic DCS can increase muscle tone, it has the potential to amplify muscle tone in conditions in which muscle tone is abnormally low (e.g., Down's syndrome). However, the question remains of how short-term effects of trans-spinal DCS on muscle tone could be transformed into long-term effects without reducing efficacy. In addition, the safety limit remains to be established.

The homeostatic signaling system acts throughout the central and peripheral nervous systems to stabilize neural function (Davis, 2013). Altering the level of neural activity results in compensatory changes in the spinal cord (Gonzalez-Islas and Wenner, 2006). Because trans-spinal DCS changes the level of neural activity, it is important to understand how trans-spinal DCS interacts with neural homoeostatic signaling systems. Answering these questions is critical for the chronic application of trans-spinal DCS.

\section{References}

Adams MM, Hicks AL (2005) Spasticity after spinal cord injury. Spinal Cord 43:577-586. CrossRef Medline

Agnew WF, McCreery DB, Yuen TG, Bullara LA (1989) Histologic and physiologic evaluation of electrically stimulated peripheral nerve: considerations for the selection of parameters. Ann Biomed Eng 17:39-60. CrossRef Medline

Ahmed Z (2011) Trans-spinal direct current stimulation modulates motor cortex-induced muscle contraction in mice. J Appl Physiol 110:14141424. CrossRef Medline

Ahmed Z (2013a) Electrophysiological characterization of spino-sciatic and cortico-sciatic associative plasticity: modulation by trans-spinal direct current and effects on recovery after spinal cord injury in mice. J Neurosci 33:4935-4946. CrossRef Medline

Ahmed Z (2013b) Effects of cathodal trans-spinal direct current stimulation on mouse spinal network and complex multijoint movements. J Neurosci 33:14949-14957. CrossRef Medline

Ahmed Z, Wieraszko A (2012) Trans-spinal direct current enhances corticospinal output and stimulation-evoked release of glutamate analog, D-2,3-(3)H-aspartic acid. J Appl Physiol 112:1576-1592. CrossRef Medline

Barron DH, Matthews BH (1938) The interpretation of potential changes in the spinal cord. J Physiol 92:276-321. Medline

Bennett DJ, Li Y, Harvey PJ, Gorassini M (2001) Evidence for plateau potentials in tail motoneurons of awake chronic spinal rats with spasticity. J Neurophysiol 86:1972-1982. Medline

Bose P, Parmer R, Thompson FJ (2002) Velocity-dependent ankle torque in rats after contusion injury of the midthoracic spinal cord: time course. J Neurotrauma 19:1231-1249. CrossRef Medline

Burke D, Wissel J, Donnan GA (2013) Pathophysiology of spasticity in stroke. Neurology 80 [3 Suppl 2]:S20-S26. CrossRef Medline

Cope TC, Bodine SC, Fournier M, Edgerton VR (1986) Soleus motor units in chronic spinal transected cats: physiological and morphological alterations. J Neurophysiol 55:1202-1220. Medline

Davis GW (2013) Homeostatic signaling and the stabilization of neural function. Neuron 80:718-728. CrossRef Medline
Eccles JC, Kostyuk PG, Schmidt RF (1962) The effect of electric polarization of the spinal cord on central afferent fibres and on their excitatory synaptic action. J Physiol 162:138-150. Medline

Elbasiouny SM, Moroz D, Bakr MM, Mushahwar VK (2010) Management of spasticity after spinal cord injury: current techniques and future directions. Neurorehabil Neural Repair 24:23-33. CrossRef Medline

Fuortes MG (1954) Direct current stimulation of motoneurones. J Physiol 126:494-506. Medline

Gilman S (1969) The mechanism of cerebellar hypotonia. An experimental study in the monkey. Brain 92:621-638. CrossRef Medline

Gonzalez-Islas C, Wenner P (2006) Spontaneous network activity in the embryonic spinal cord regulates AMPAergic and GABAergic synaptic strength. Neuron 49:563-575. CrossRef Medline

Gracies JM, Nance P, Elovic E, McGuire J, Simpson DM (1997) Traditional pharmacological treatments for spasticity. Part II: General and regional treatments. Muscle Nerve [Suppl 6]:S92-S120. Medline

Heckmann CJ, Gorassini MA, Bennett DJ (2005) Persistent inward currents in motoneuron dendrites: implications for motor output. Muscle Nerve 31:135-156. CrossRef Medline

Hornby TG, Rymer WZ, Benz EN, Schmit BD (2003) Windup of flexion reflexes in chronic human spinal cord injury: a marker for neuronal plateau potentials? J Neurophysiol 89:416-426. CrossRef Medline

Kapitza S, Zörner B, Weinmann O, Bolliger M, Filli L, Dietz V, Schwab ME (2012) Tail spasms in rat spinal cord injury: changes in interneuronal connectivity. Exp Neurol 236:179-189. CrossRef Medline

Katz RT, Rymer WZ (1989) Spastic hypertonia: mechanisms and measurement. Arch Phys Med Rehabil 70:144-155. Medline

Lance JW (1980) Spasticity: disordered motor control. In: Symposium synopsis (Feldman RG, Young RR, Koella WP, eds). Chicago: Year Book Medical Publishers.

Marsala M, Hefferan MP, Kakinohana O, Nakamura S, Marsala J, Tomori Z (2005) Measurement of peripheral muscle resistance in rats with chronic ischemia-induced paraplegia or morphine-induced rigidity using a semiautomated computer-controlled muscle resistance meter. J Neurotrauma 22:1348-1361. CrossRef Medline

Marsan CA, Fuortes MG, Marossero F (1951) Effects of direct currents on the electrical activity of the spinal cord. J Physiol 113:316-321. Medline

Matthews PBC (1972) Mammalian muscle spindles and their central actions. London: Arnold.

Merrill DR, Bikson M, Jefferys JG (2005) Electrical stimulation of excitable tissue: design of efficacious and safe protocols. J Neurosci Methods 141: 171-198. CrossRef Medline

Onushko T, Hyngstrom A, Schmit BD (2011) Bilateral oscillatory hip movements induce windup of multijoint lower extremity spastic reflexes in chronic spinal cord injury. J Neurophysiol 106:1652-1661. CrossRef Medline

Roy RR, Edgerton VR (2012) Neurobiological perspective of spasticity as occurs after a spinal cord injury. Exp Neurol 235:116-122. CrossRef Medline

Shumway-Cook A, Woollacott MH (1985) Dynamics of postural control in the child with Down syndrome. Phys Ther 65:1315-1322. Medline

Subramony SH, Ashizawa T (1993) Spinocerebellar ataxia type 1. In: GeneReviews (Pagon RA, Adam MP, Bird TD, Dolan CR, Fong CT, Stephens K, eds). Seattle WA: University of Washington. Available at http://www.ncbi.nlm.nih.gov/books/NBK1184/.

Takakusaki K (2008) Forebrain control of locomotor behaviors. Brain Res Rev 57:192-198. CrossRef Medline

Taylor JS, Friedman RF, Munson JB, Vierck CJ Jr (1997) Stretch hyperreflexia of triceps surae muscles in the conscious cat after dorsolateral spinal lesions. J Neurosci 17:5004-5015. Medline

Thompson FJ, Browd CR, Carvalho PM, Hsiao J (1996) Velocity-dependent ankle torque in the normal rat. Neuroreport 7:2273-2276. Medline

Yates CC, Charlesworth A, Reese NB, Ishida K, Skinner RD, Garcia-Rill E (2009) Modafinil normalized hyperreflexia after spinal transection in adult rats. Spinal Cord 47:481-485. CrossRef Medline 